\title{
Optimization Design of Stiffener for Buckling in the Cylindrical Structure Ri Zhang ${ }^{1,}$, Chengzhong $\mathrm{Qu}^{1}$, Qiang Guo², Ling lu ${ }^{3}$, \\ ${ }^{1}$ School of Architecture, Northeast Dianli University, Jilin 132012, China \\ ${ }^{2}$ Changchun Passenger Car Co., LTD, China Railway Rolling Stock Corporation, Changchun 130000, China \\ ${ }^{3}$ Economic\&Technology Research Institute, State Grid Shandong Electric Power Company, Jinan 250000, China \\ azhangri2007@163.com
}

Keyword: Cylindrical Structure, Stiffener for Buckling, Optimization

\begin{abstract}
This article is based on zero order optimization method, proposed a method for optimization design of stiffener in the cylindrical structure. Use APDL to make a computer program to solve this problem. There is a series of work in this program including modeling, loading, post processing and optimization. This method makes the progress of optimization design about stiffener for buckling easy.
\end{abstract}

\section{Introduction}

Cylindrical structure is a very important structure method in civil engineering. Mechanical properties of cylindrical structures are very perfect. During the application, the cylindrical structure is easy to buckling. Many domestic and foreign scholars have made a great deal of researches about the buckling formula of cylindrical structure [1], to make the cylindrical structure hard to buckling, we sometimes increase the thickness of wall of the cylindrical structure or adding stiffener to cylindrical structure. There is no perfect buckling theory of cylindrical structure with stiffener, this paper proposes a method based on APDL. First, known to the height of the cylindrical structure, thickness of cylindrical structure wall, the radius of the cylindrical structure and the buckling performance requirements, according to the data above, build Finite Element Model in ansys, automatic meshing without any fault, calling OPT module using APDL, variables could be optimized by zero order optimization method, provide core data for user to choose from, the whole process is automatic, making the process more simple and saving a lot of time.

\section{Factor of Resistance to Buckling}

In the process of optimization, we must evaluate the optimization results, this paper aims at finding the most suitable result. So we put forward factor of resistance to buckling, it can be abbreviated to RBF, RBF is optimal object. First, in this paper modeling the cylindrical structure without stiffener in ansys [2,3], according to user's requirements imposed the load, call buckling analysis module in ansys, extract buckling eigenvalue named eng1. Second, modeling the cylindrical structure with stiffener, extract buckling eigenvalue named eng2 after buckling analysis, then get value of the stiffener width named width, get value of the stiffener thickness named thickness, the number of stiffener N, then we define the RBF with the follow Eq.1.

$$
R B F=N \times \text { width } \times \text { thinckness } \div(\text { eng } 1 \text {-eng } 2)
$$

In the design process of the stiffener, the smaller the RBF, the better the size of stiffener, RBF is the objective function of optimization.

\section{Optimization Method}

Using the zero order function to complete the optimization [4], dependent variable by the least 
squares fitting value approximation, constrained minimization problem is transformed into unconstrained problem by penalty function, the minimization process in iterative approximation of penalty function [5].

$$
\hat{f}(X)=f(X)+\varepsilon_{f}
$$

Eq. 3, Eq. 4 , Eq. 5 is state variable

$$
\begin{aligned}
& \hat{g}(X)=g(X)+\varepsilon_{g} \\
& \hat{h}(X)=h(X)+\varepsilon_{h} \\
& \hat{w}(X)=w(X)+\varepsilon_{w}
\end{aligned}
$$

Eq. 6 as a quadratic polynomial form

$$
\hat{f}=a_{0}+\sum_{i}^{n} a_{i} x_{i}+\sum_{i}^{n} \sum_{j}^{n} b_{i j} a_{i} x_{j}
$$

According to the approximate, equation can be represented as follow

$$
\begin{aligned}
& \min \hat{f}(X)=\hat{f}(X) \\
& x_{i l} \leq x_{i} \leq x_{h}(i=1,2,3, \ldots n) \\
& \hat{g}(X) \leq \hat{g}_{i h}+a_{i}\left(i=1,2,3, \ldots m_{1}\right) \\
& h_{i l}-\beta_{i} \leq \hat{h}(X)\left(i=1,2,3, \ldots m_{2}\right) \\
& w_{i l}-r_{i} \leq \hat{w}_{i}(X) \leq w_{i h}+r_{i}\left(i=1,2,3, \ldots m_{3}\right)
\end{aligned}
$$

After above progress, constrained minimization problem is transformed into unconstrained problem as Eq. 12

$$
\min F\left(X, p_{k}\right)=\hat{f}+f_{0} p_{k}\left[\sum_{i=1}^{n} X\left(x_{i}\right)+\sum_{i=1}^{m_{1}} G\left(\hat{g}_{i}\right)+\sum_{i=1}^{m_{2}} H\left(\hat{h}_{i}\right)+\sum_{i=1}^{m_{3}} W\left(\hat{w}_{i}\right)\right]
$$
14

In this paper, independent variable is width, thickness, $\mathrm{N}$, constraint equation is as Eq. 13 and Eq.

$$
\begin{aligned}
& (\text { eng } 1 \text {-eng } 2) \geq 0 \\
& (\text { eng } 1 \text {-eng } 2) \div \text { eng } 2 \geq 0
\end{aligned}
$$

BPI is buckling performance improvements in percentage

\section{Case}

A case study of optimization of wind power tower, in this case, the height of the wind tower is $60 \mathrm{~m}$, radius is $1.5 \mathrm{~m}$, thickness of wall is 0.025 meters, horizontal load and vertical load on the top of it, vertical load is $1700 \mathrm{KN}$, horizontal load is $440 \mathrm{KN}$. Stiffener is evenly distributed around the 
inner of wind tower, the height of the stiffener is in the range of $1 \mathrm{~m}$ to $30 \mathrm{~m}$, the width of the stiffener is in the range of $0.005 \mathrm{~m}$ to $0.035 \mathrm{~m}$, the thickness of the stiffener is in the range of $0.1 \mathrm{~m}$ to $0.35 \mathrm{~m}$. the number of stiffener $\mathrm{N}$ is distributed variable. They are $10,13,16,19,22,25$, respectively, BPI is $10 \%, 20 \%, 30 \%, 40 \%, 50 \%, 60 \%$ respectively. Fig. 1 shows the shape of the model.

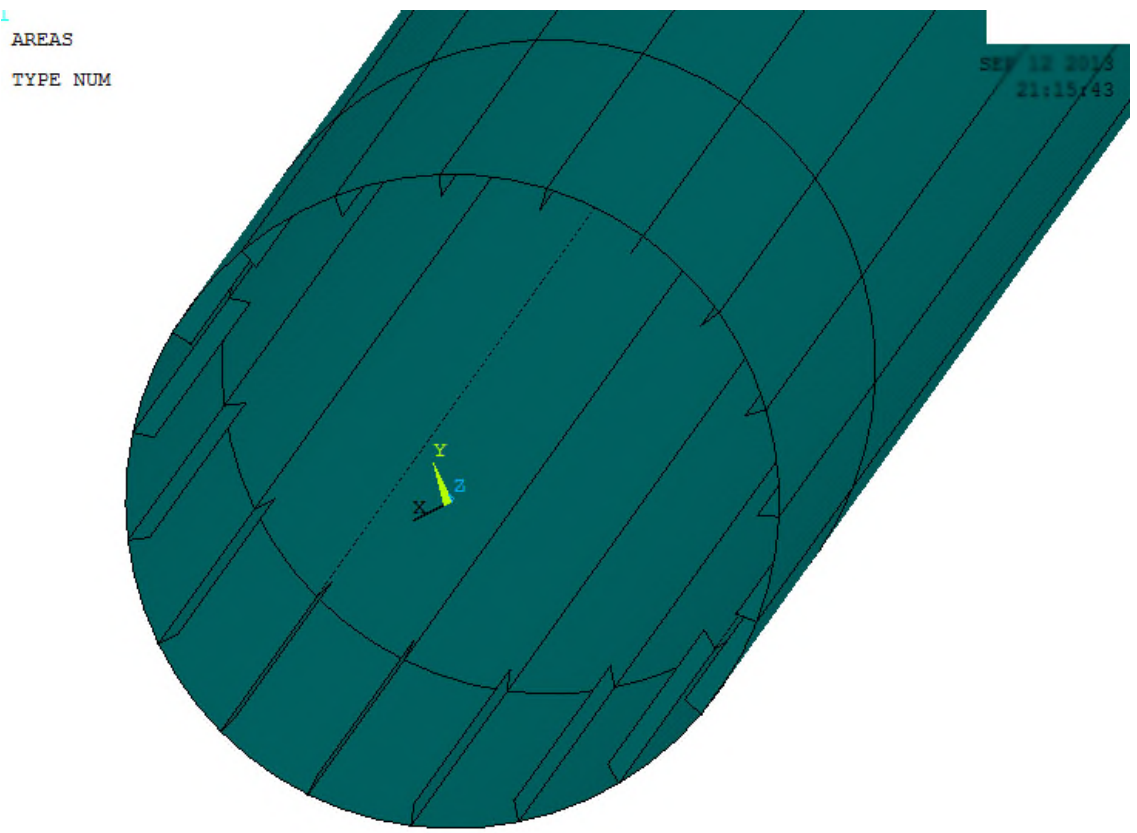

Fig. 1 Model.

Based on the given conditions, computer calls programs already be written, the computer automatic calculate the size of the stiffener.

Limitation of BPI. The results show that, when $\mathrm{N}$ is constant, the value of BPI is limited, Fig. 2 shows the results, $\mathrm{x}$-axis is $\mathrm{N}$ (the number of stiffener), $\mathrm{y}$-axis is BPI, different $\mathrm{N}$ corresponds to different BPI, BPI increases with $\mathrm{N}$.

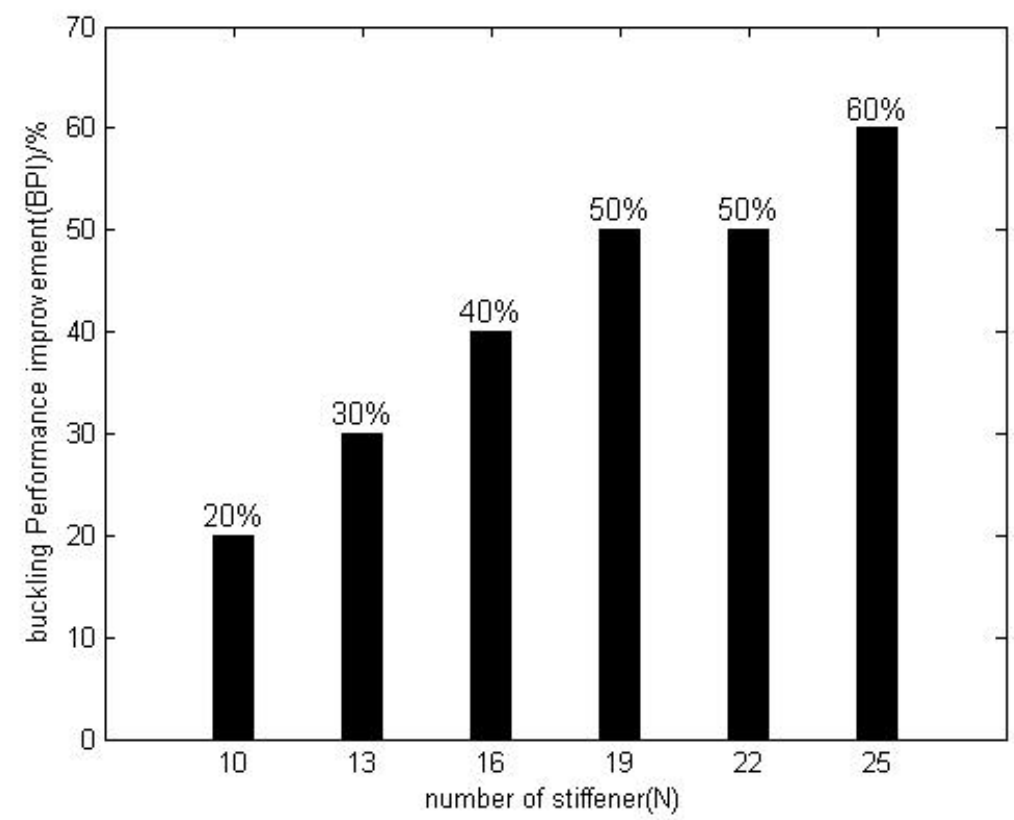

Fig. 2 Limitation of BPI.

Optimization of Height. After the optimization, the program find the best height of stiffener, when $\mathrm{N}$ and BPI is a constant, it shows in Table 1 
Table 1. Results of optimization about height.

\begin{tabular}{ccccccc}
\hline Height $[\mathrm{m}]$ & 10 & 13 & 16 & 19 & 22 & 25 \\
\hline $10 \%$ & 3.6084 & 3.1052 & 2.7357 & 3.055 & 2.8485 & 2.9598 \\
$20 \%$ & 6.8608 & 6.3084 & 6.3055 & 11.012 & 10 & 6.7842 \\
$30 \%$ & null & 9.6454 & 9.5686 & 10.507 & 9.2253 & 11.899 \\
$40 \%$ & null & null & 12.363 & 13.69 & 14.604 & 12.175 \\
$50 \%$ & null & null & null & 14.34 & 15.255 & 15.396 \\
$60 \%$ & null & null & null & null & null & 18.403 \\
$70 \%$ & null & null & null & null & null & null \\
\hline
\end{tabular}

$\mathrm{X}$-axis is $\mathrm{N}$ (the number of stiffener), $\mathrm{y}$-axis is the height of stiffener. Fig. 3 shows polyline with different BPI. We conclude that the height remains fairly unchanged as the $\mathrm{N}$ changes, to make it clearer, we focus on BPI $=10 \%$, Fig. 4 shows that the height of stiffener fluctuates within $10 \%$ with the $\mathrm{N}$ changes.

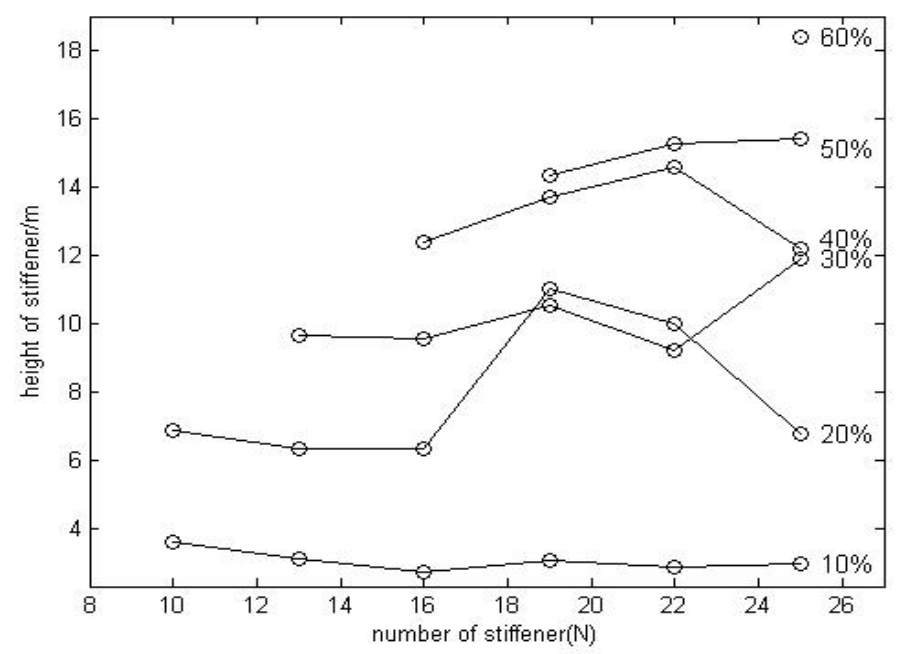

Fig. 3 Height in different BPI.

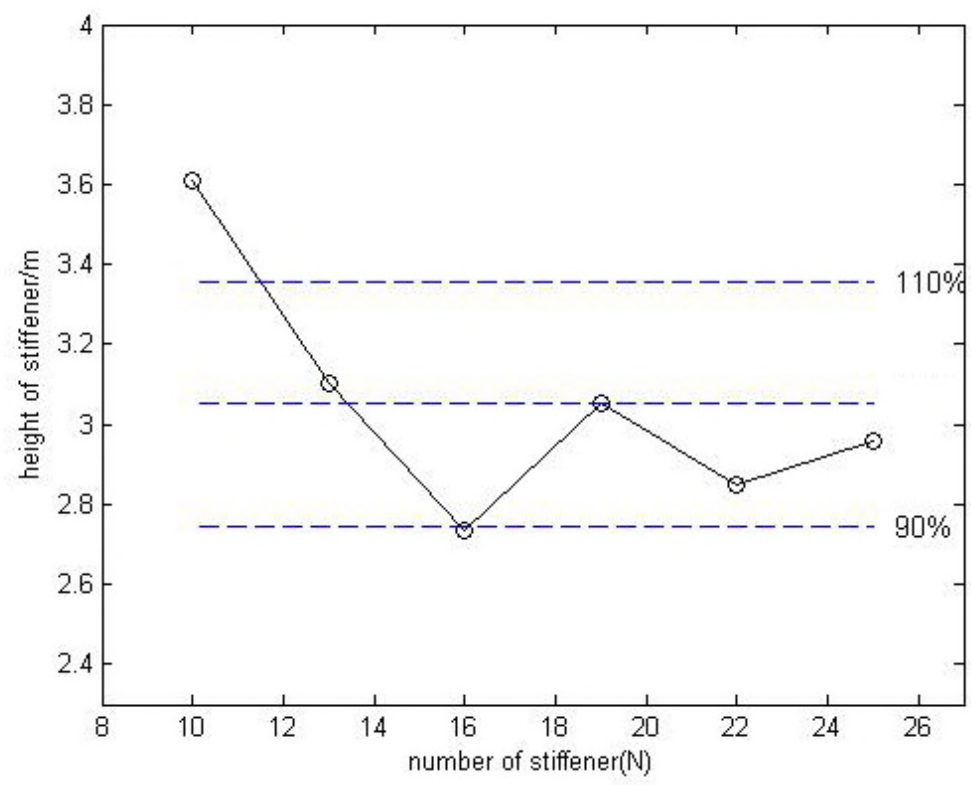

Fig. 4 BPI=10\%.

When $\mathrm{N}$ and BPI is a constant, the relationship between height and BPI shows in Fig. 5, $\mathrm{x}$-axis is $\mathrm{BPI}, \mathrm{y}$-axis is the height of stiffener, polyline with different $\mathrm{N}$ is showed in Fig. 5, we come to conclusion that the height is in proportion to the BPI. 


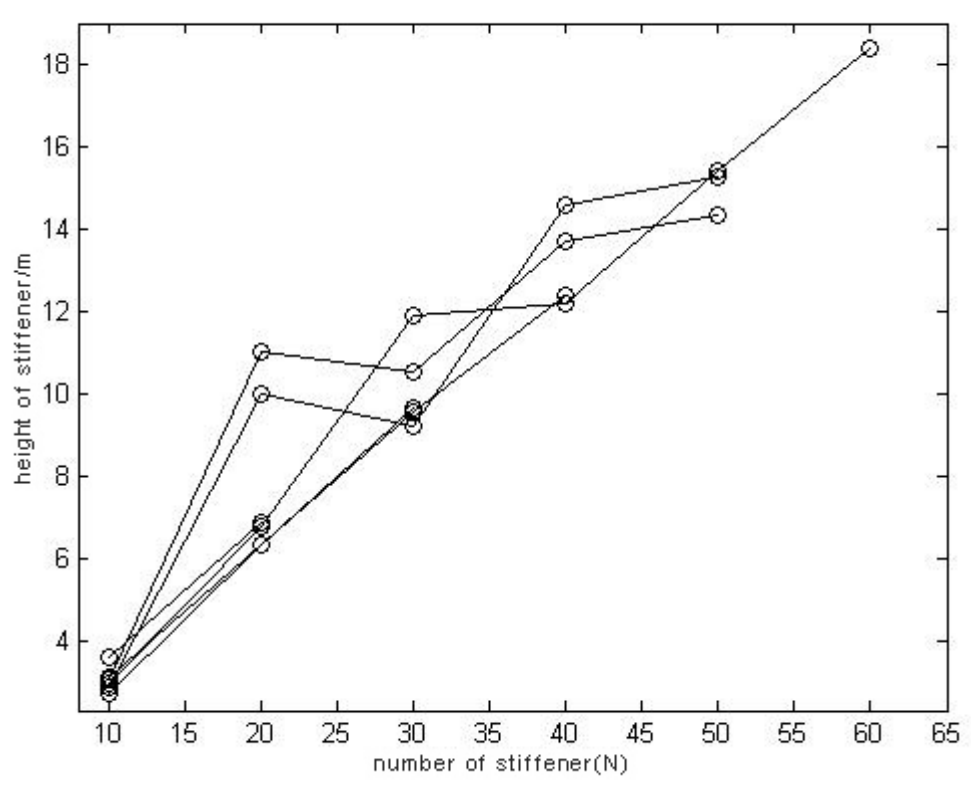

Fig. 5 Relationship BPI and N.

\section{Summary}

This article shows intelligent design of the stiffener, this method make the whole process more convenient, and this paper shows the limitation of buckling performance with different number of stiffener, find the relationship of buckling performance and the height of the stiffener.

\section{References}

[1] C. Fleury, Structural steelwork Analysis safety against Buckling of shells DIN18800 Part 4, J. Struct. Eng. 1990

[2] M. Saeed, Finite Element Analysis Theory and Application with ANSYS. Prentice Hall, USA, 2003.

[3] S. E. Kim, W. F. Chen, Practical advanced analysis for unbraced steel frames design, J. Struct. Eng. 122(11) (1996) 1259-1265.

[4] Z. Quan, Z. Deming, Integral global minimization: Algorithms, implementations and numerical tests. J. Global Optim. 7(4) (1995) 421-454.

[5] C. Fleury. First and second order convex approximation strategies in structural optimization. Struct. Optim. 1(1) (1989) 3-10. 